\title{
DISPERSION BEHAVIOR OF CATIONIC SURFACTANT TREATED REDUCED GRAPHENE OXIDE IN DIFFERENT SOLVENTS
}

\author{
K.N. Amba Sankar ${ }^{1}$, P. Nandakumar ${ }^{1,}$, C. Sathishkumar ${ }^{2}$, R. Deepa ${ }^{3}$ \\ and Kallol Mohanta ${ }^{4}$ \\ ${ }^{1}$ Department of Electronics, PSG College of Arts \& Science, Coimbatore-641014, Tamilnadu. \\ ${ }^{2}$ Department of Chemistry, Sardar Vallabhbhai Patel International School of Textiles and \\ Management, Coimbatore-641004, Tamil Nadu, India \\ ${ }^{3}$ Department of Information Technology, PSG College of Arts \& Science, Coimbatore-641014. \\ ${ }^{4}$ Nanotech Research Innovation and Incubation Centre (NRIIC), PSG Institute of Advanced \\ Studies, Coimbatore- 641004, Tamil Nadu, India \\ ${ }^{\bowtie}$ Corresponding Author: nandacjb@gmail.com
}

\begin{abstract}
The dispersion behavior of chemically modified reduced graphene oxide (CRGO) has been investigated in five different solvents. Herein, we used cationic surfactant CTAB as a dispersing agent to prevent the agglomeration of graphene layers. The main role of the surfactant is to create dangling bonds on graphene layers and those help to exfoliate. In the physical process, ultra-sonication is used to break the $\pi$ bonds whereas, in the chemical process, surfactant molecules occupy the space between the graphene layers. The dispersion behavior of CRGO in different liquid media has been observed for over a month. The chemically reduced graphene oxide was characterized by Transmission electron microscopy (TEM) analysis and current-voltage (I-V) measurement. Keywords: CRGO, Organic Solvents, Dispersion Stability, TEM, I-V Characterization
\end{abstract}

RASĀYAN J. Chem., Vol. 14, No.4, 2021

\section{INTRODUCTION}

In recent decades conductive inks are widely investigated due to potential applications in flexible and printed electronics. ${ }^{1-9}$ Generally, conductive coatings are used to cover dielectric substrate and increase electron mobility in less conductive films. ${ }^{10,11}$ Conductive metallic nanomaterials ${ }^{12}$ and carbon-based $0 \mathrm{D}$, $1 \mathrm{D}$, and $2 \mathrm{D}$ materials ${ }^{13}$ ensure potential applications in growing scientific areas. These kinds of nanomaterials have good transparency and conducting behavior and are used in advanced electronic device applications such as transparent electrodes ${ }^{14-18}$, electromagnetic interference shielding ${ }^{19}$, solar cell, liquid crystal display, supercapacitor, lubricant systems ${ }^{20}$, and smart textiles. ${ }^{21-23}$

Graphene is a 2D nanostructured carbon allotrope with a high surface area. It has unique electrical, strong mechanical, and good thermal properties. Graphene cleaves to immense assure for fabricating a choice of electronic devices, for example, energy production and storage, conducting reinforcing addition materials, sensors, transparent electrodes, and so on. The unique properties of graphene come from the individually separated layers, but isolated graphene layers tend to agglomerate together as a result of van der Waals force exhibited from $\pi-\pi$ hoarding; then it forms the graphitic structure. These appearances create graphene mainly beneficial in energy expertise. In addition, graphene can be shifted to substrates for clear electronic applications that permit the production of crystal clear or semi-transparent energy-producing and storage devices. To avoid the agglomeration of graphene layers, various approaches like surface modification through ultrasonic and microwave processes which come under physical dispersion and chemical modifications using polymer composites and organic coatings have been executed. These techniques offer a capable slant for the fabrication of new electronic devices. ${ }^{24-29}$

For graphene synthesis mainly two approaches such as wet chemical and non-wet chemical methods are followed. The non-wet chemical method gives high quality but has a low yield, on the other hand, the wet 
chemical method is a scalable process as the yield is high. In wet chemical processes, chemical modification and non-covalent functionalization play important roles in surface modification at edges and on graphene layers. It improves the solubility or dispersion stability in organic solvents. ${ }^{30}$

Generally, surface modifications on graphene have been employed to make stable dispersion in an aqueous medium and different organic solvents or surfactants. ${ }^{31,32}$ The surface modification is created by chemical modification and non-covalent functionalization. In the chemical modification process, the CTAB creates bonding on graphene layers. ${ }^{33}$

The three main stages to make perfect graphene layers through the wet chemical method are oxidation, intercalation, and reduction process. In the oxidation process, chemical exfoliation is done via a strong oxidation agent to split the $\pi$ bond, and the force of this method to the carboxyl and hydroxyl functional groups are founded around the exfoliated graphite coatings to modify the structural arrangement of the layers transformed it to graphene oxide. ${ }^{34,35}$ The intercalation process helps to prevent agglomeration in reduced graphene oxide and finally reduction process is carried out to remove the 'oxide' components to gives few-layer graphene; but due to electrostatic force, it may possible that re-agglomeration can happen. On the other side, in non-covalent functionalization, sulphonate groups create modifications on graphene layers. Non-covalent functionalized graphene prevents stacking in $\pi$ conjugated systems and makes them isolated. But the chemical modification of graphene layers or embedding them into polymer matrix affects its electrical property due to the attachment of organic radicals.

In this work, we explained the synthesis process of cationic surfactant treated reduced graphene oxide and compare the dispersion behavior in five different solvents i.e. DI water, acetone, ethanol, chloroform, and methanol. The cationic surfactant plays an important role in stable dispersion behavior via surface modification around the graphene layer. It prevents the agglomeration of graphene layers due to steric force.

\section{Material and Methods}

\section{EXPERIMENTAL}

Natural graphite powder of 60 mesh size, methanol, acetone, hydrochloric acid $(\mathrm{HCl})$, Hydrogen peroxide $\left(\mathrm{H}_{2} \mathrm{O}_{2}\right)$, and Sulfuric acid $\left(\mathrm{H}_{2} \mathrm{SO}_{4}\right)$ was purchased from Loba Chem, Potassium permanganate $\left(\mathrm{KMnO}_{4}\right)$ was purchased from Merck, Sodium nitrate $\left(\mathrm{NaNO}_{3}\right)$ was purchased from SDFCL, CTAB and chloroform were purchased from Sigma Aldrich. Ethanol was purchased from Changshu Longsheng Fine Chemical Co. Ltd. Millipore water was used for all the experiments, and Current-Voltage (I-V) curve was taken by Keithley Source Meter 2450.

\section{General Procedure}

Graphene oxide (GO) be prepared via the adapted Hummer's method. ${ }^{36}$ The synthesis process of GO and CRGO was reported elsewhere. The chemical oxidation process was used to synthesize graphene oxide from natural graphite powder. In this process, concentrated $\mathrm{H}_{2} \mathrm{SO}_{4}, \mathrm{KMnO}_{4}$, and $\mathrm{NaNO}_{3}$ were used for oxidizing the graphite flakes. After the chemical exfoliation process, we get a mixture of graphene and few graphite oxide flakes in the form of a homogeneous solution. In the filtering process - low-speed centrifugation at around $900 \mathrm{rpm}$ separates graphite oxide and it settles at bottom of the centrifuge tube, and at high-speed centrifugation around $6000 \mathrm{rpm}$ removes the unwanted molecular ingredients. In CRGO synthesis, we used cationic surfactant CTAB to modify the surface of graphene layers through chemical techniques instead of the ultrasonication and reduction process. ${ }^{37}$

\section{Synthesis of Surface Modification in Graphene Sheets Via Ionic Interaction}

In the chemical reduction process, centrifugation takes the main role in the filtering process to get the few layers CRGO - at low-speed centrifugation around $900 \mathrm{rpm}$ to separate the large graphite particles and after that high speed $8000 \mathrm{rpm}$ centrifugation of the supernatant to remove the excess surfactant and reducing compounds from $\mathrm{CRGO} .{ }^{38}$

\section{Characterization}

The morphology study was conceded out throughout High-Resolution Transmission Electron Microscope (HRTEM) by JEOL JEM 2100 as well as the electrical description of CRGO thin-film conceded out by Keithley Source Meter 2450 utilizing two probe techniques. 


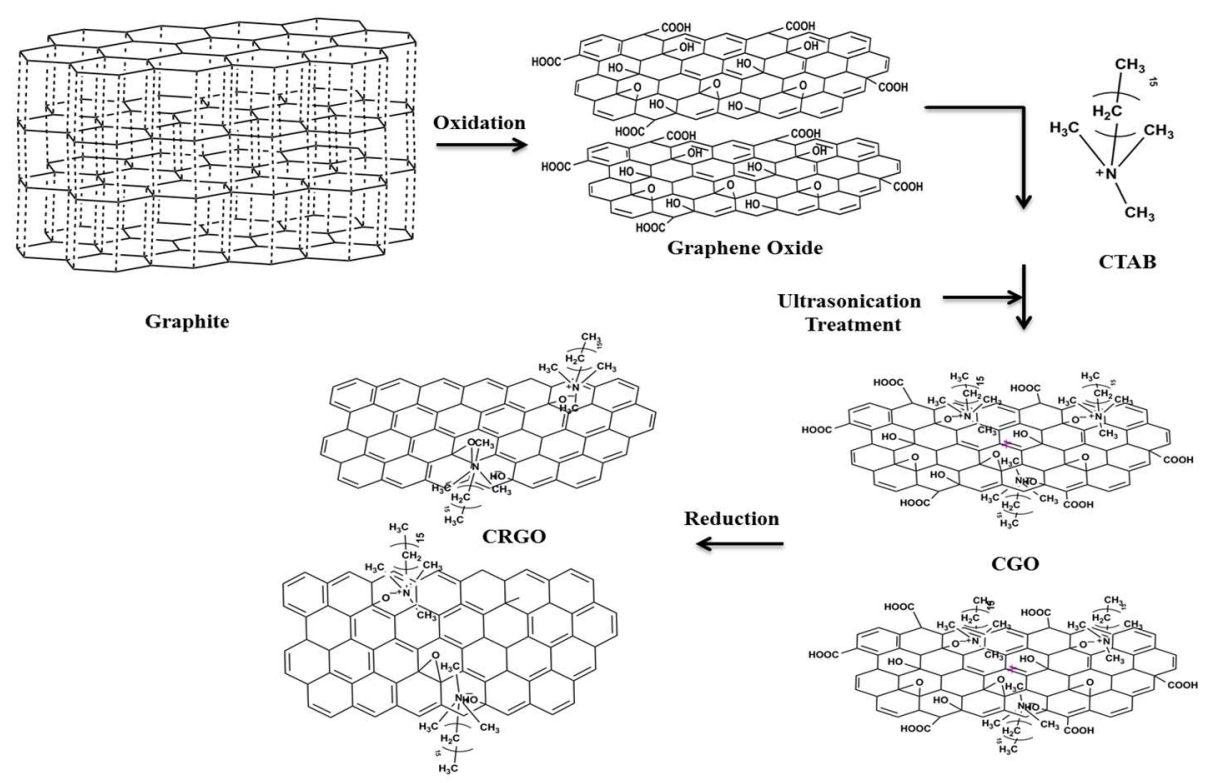

Fig.-1: Schematic Representation of CRGO Synthesis Process

\section{RESULTS AND DISCUSSION}

In our preceding paper, we conversed the synthesis procedure of CRGO and inveterate it with Fourier Transformed Infra-Red spectra, X-Ray Diffraction patterns and Transmission Electron Microscope images and dispersion stability in an aqueous medium. In this effort, we clarified the dispersion stability of CRGO in dissimilar solvents such as deionized water $\left(\mathrm{H}_{2} \mathrm{O}\right)$, methanol $\left(\mathrm{CH}_{3} \mathrm{OH}\right)$, acetone $\left(\mathrm{C}_{3} \mathrm{H}_{6} \mathrm{O}\right)$, ethanol $\left(\mathrm{C}_{2} \mathrm{H}_{5} \mathrm{OH}\right)$, and chloroform $\left(\mathrm{CH}_{3} \mathrm{Cl}\right)$ were employed as dispersion media.

Graphene is an n-type material. It has localized quasi-free electrons on specific moieties of individual sheets. These moieties share their electrons with the moieties of another graphene sheet when they are stacked on each other and try to create aggregations. This is called $\pi-\pi$ stacking interaction also a weak van der Walls force comes into play due to shifting electronic charge density.

The electrostatic force and van der Walls force (steric forces) are strong enough to agglomerate the layers of the graphene oxide again and again unless the layers are well separated and functionalized with cationic groups provided by CTAB. Thus, a long ( $\sim 8 \mathrm{~h}$ with breaks in between) sonication is necessary (a) to break the weak van der Waals force by using the liquid exfoliation method and (b) to make a stable supernatant suspension homogeneous CRGO solution via long time sonication process

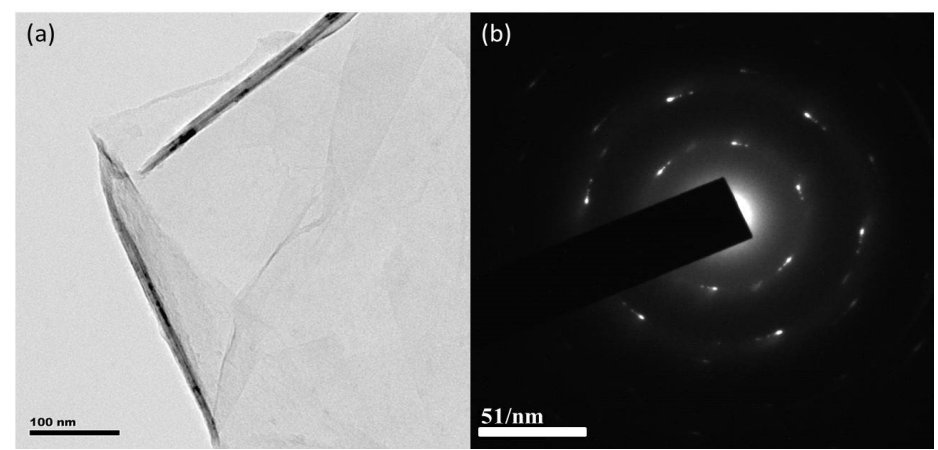

Fig.-2: Picture of (a) HRTEM and (b) Selected Area Electron Diffraction Pattern of CRGO

Figure- 2 shows that the morphological results of CRGO carried out by HRTEM characterizations. The thin CRGO layers with large magnification $(100 \mathrm{~nm})$ could be seen in the HRTEM image. In Figure 2(b), the selected area energy dispersion (SAED) patterns clearly show the hexagonal spots which reveal the crystallinity of the CRGO. 
RASĀYAN J. Chem.

Vol. 14 | No. 4 |2196-2201| October- December | 2021

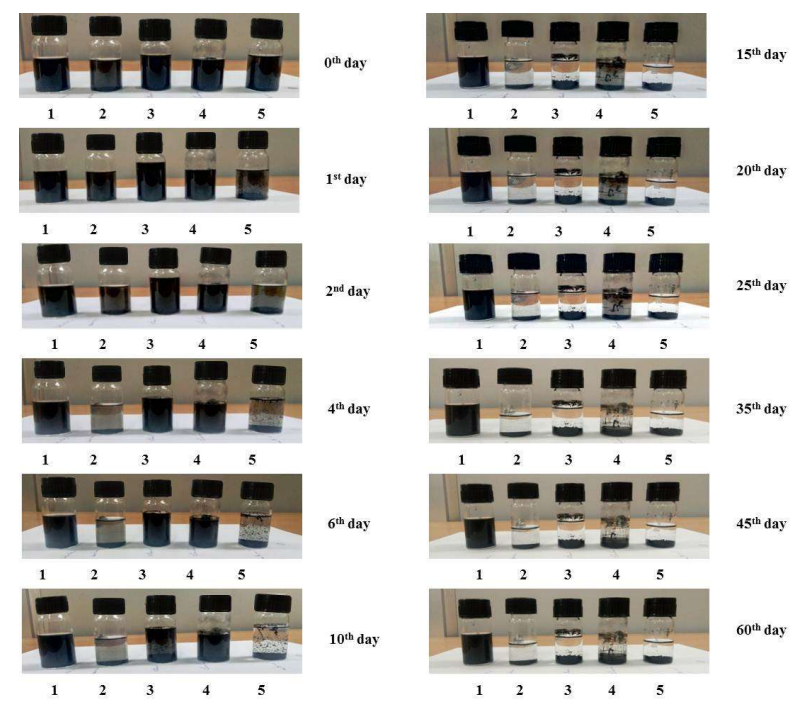

Fig.-3: Dispersion Stability of Synthesized CRGO dispersed in Different Solvents (1- DI water, 2- acetone, 3ethanol, 4- chloroform, and 5- methanol) and the Digital Picture was taken Different Time Durations.

The dispersion behavior of CRGO has been investigated at a concentration of $0.2 \mathrm{mg} \mathrm{ml}-1$ in different solvents. Digital pictures were taken to display the dispersion quality of CRGO in five different solvents without any disturbance from 0 to 60 days' time duration at room temperature.

After one week, CRGO showed a stable dispersion in water, ethanol, and chloroform. In other solvents, CRGO flakes were started to sediment. From the 2nd day onwards dispersed CRGO particles in methanol solvent had started to settle slowly and on the 10th day, nearly all the particles had settled at the bottom of the vial. In acetone, dispersed particles had been agglomerated and started settle on day 2. Furthermore, all CRGO particles had settled at the bottom on day 15 but the other three different solvents, flakes remained dispersed for up to a week. But from the 10th day onwards particles were started to settle in ethanol solvent and on day 15th particles were completely settled at the bottom. In chloroform, most of the particles were settled within day 20. But in the water, there was no sedimentation and flocculation even after storing the vial for more than 60 days. Thus, CRGO dispersion in water exhibited long term stability

\section{Current-Voltage (I-V) Characterization of CRGO Film}

The synthesized CRGO solution was applied on the substrate via drop-casting technique and currentvoltage (I-V) characterizations were measured. The CRGO conductive ink was obtained with $20 \mathrm{mg}$ ml-1 of CRGO and $10 \mathrm{wt} \%$ of PVA in ethanol. The mixture solution was applied on $1 \mathrm{x} 1 \mathrm{~cm} \mathrm{Al}_{2} \mathrm{O}_{3}$ substrate using the drop-casting technique and the aluminum electrodes were cleanly deposited with thermal evaporation technique. The electrical properties were analyzed using two probe electrode methods.
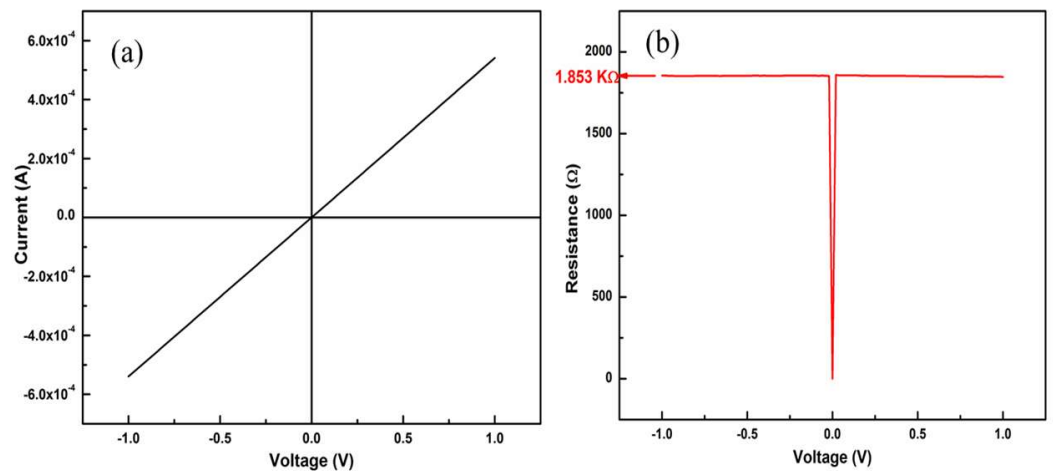

(c)

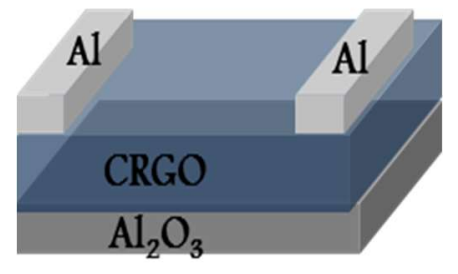

Fig.-4: (a) Current-voltage (I-V) Characteristics, (b) Resistance Value of CRGO Film and (c) Schematic of CRGO Film with Contact Electrodes 
RASĀYAN J. Chem.

Vol. 14 | No. 4 |2196-2201| October- December | 2021

Figure 4 (a) shows the I-V graph of drop-casted CRGO coated films $1 \mathrm{~cm} 2$ size taken from a voltage range of -1 to $+1 \mathrm{~V}$. The current measure shows a maximum of $0.53 \mathrm{~mA}$ at $1 \mathrm{~V}$ and (b) shows the resistance value CRGO film value around $1853 \Omega$. We painted a line on a paper sheet with concentrated CRGO ink, it showed a sheet resistance value around $1.329 \mathrm{M} \Omega \mathrm{cm}^{-1}$.

\section{CONCLUSION}

In summary, the dispersion behaviors of CRGO in five different solvents were compared. The $\mathrm{H}_{2} \mathrm{O}$ solvent showed excellent dispersion stability and homogeneous solution for more than a month of period compare to the other four solvents. Surface modification and sonication were the main roles played in the dispersion behavior of CRGO. The surface modification created by two main factors was the ultrasonication process to break the weak van der Waals and surfactant creates steric force it prevents agglomeration. The CRGO film resistance value $1834 \Omega$ was obtained.

\section{REFERENCES}

1. Dan Li, Marc B. Müller, Scott Gilje, Richard B. Kaner, and Gordon G. Wallace, Nature Nanotechnology, 3(2), 101(2008), http://doi.org/10.1038/nnano.2007.451

2. J. I. Paredes, S. Villar Rodil, A. Martínez Alonso, and J. M. D. Tascón, Langmuir, 24(19), 10560(2008), http://doi.org/10.1021/la801744a

3. John Texter, Current Opinion in Colloid \& Interface Science, 19(2), 163(2014), http://doi.org/10.1016/j.cocis.2014.04.004

4. Dimitrios Konios, Minas M.Stylianakis, Emmanuel Stratakis, and Emmanuel Kymakis, Journal of Colloid and Interface Science, 430, 108(2014), http://doi.org/10.1016/i.jcis.2014.05.033

5. Rana Tariq Mehmood, Seung HoHong, Tian ZiShen, and Jang KunSong, Carbon, 98, 188(2016), http://doi.org/10.1016/j.carbon.2015.11.007

6. Devesh K. Chouhan, T. Umasankar Patro, G. Harikrishnan, Sanjay Kumar, Siddharth Gupta, Sudheer Kumar, Hagai Cohen, and H. Daniel Wagner, Applied Clay Science, 132, 105(2016), http://doi.org/10.1016/j.clay.2016.05.023

7. Xiang Ji, Yahui Song, Jing Han, Lin Ge, Xiaoxiang Zhao, Chen Xu, Yongqiang Wang, Di Wu, and Haixia Qiu, Journal of Colloid and Interface Science, 497, 317(2017), http://doi.org/10.1016/j.jcis.2016.09.049

8. Yuxi Xu, Hua Bai, Gewu Lu, Chun Li, and Gaoquan Shi, Journal of the American Chemical Society, 130(18), 5856(2008), http://doi.org/10.1021/ja800745y

9. Qunxiang Ren, Li Feng, Ronghua Fan, Xin Ge, and Yingying Sun, Materials Science and Engineering C, 68, 308(2016), http://doi.org/10.1016/j.msec.2016.05.124

10. KN Amba Sankar, Kallol Mohanta, Journal of Electronic Materials, 47(3), 1970(2018), http://doi.org/10.1007/s11664-017-5998-3

11. Siegfried Eigler, and Andreas Hirsch, Angewandte Chemie International Edition, 53(30), 7720(2014), http://doi.org/10.1002/anie.201402780

12. R Venkata Krishna Rao, K Venkata Abhinav, P.S. Karthik, and Surya Prakash Singh, RSC Advances, 5(95), 77760(2015), http://doi.org/10.1039/c5ra12013f

13. Kyoungho Min, Tae Hee Han, Joohoon Kim, Jiyoung Jung, Cheolsoo Jung, Soon Man Hong, and Chong Min Koo, Journal of Colloid and Interface Science, 383(1), 36(2012), http://doi.org/10.1016/j.jcis.2012.06.021

14. Jizhong Song, and Haibo Zeng, Angewandte Chemie International Edition, 54(34), 9760(2015), http://doi.org/10.1002/anie.201501233

15. Kallol Mohanta, M Arturo López-Quintela, José Rivas, Organic Electronics, 46, 247(2017), http://doi.org/10.1016/j.orgel.2017.04.011

16. Ethan B. Secor, Pradyumna L. Prabhumirashi, Kanan Puntambekar, Michael L. Geier and Mark C. Hersam, The Journal of Physical Chemistry Letters, 4(8), 1347(2013), http://doi.org/10.1021/jz400644c

17. Lu Huang, Yi Huang, Jiajie Liang, Xiangjian Wan, and Yongsheng Chen, Nano Research, 4(7), 675(2011), http://doi.org/10.1007/s12274-011-0123-Z 
RASĀYAN J. Chem.

Vol. 14 | No. 4 |2196-2201| October- December | 2021

18. Wendong Yang, and Changhai Wang, Journal of Materials Chemistry C, 4(30), 7193(2016), http://doi.org/10.1039/c6tc01625a

19. Pradip Kumar, Faisal Shahzad, Seunggun Yu, Soon Man Hong, Yoon Hyun Kim, and Chong Min Koo, Carbon, 94, 494(2015), http://doi.org/10.1016/j.carbon.2015.07.032

20. Ajay Chouhan, Harshal P.Mungse, and Om P.Khatri, Advances in Colloid and Interface Science, 102215(2020), http://doi.org/10.1016/j.cis.2020.102215

21. Yuning Meng, Yang Zhao, Chuangang Hu, Huhu Cheng, Yue Hu, Zhipan Zhang, Gaoquan Shi, and Liangti Qu, Advanced Materials, 25(16), 2326(2013), http://doi.org/10.1088/2515-7639/abc632

22. Yong Ju Yun, Won G. Hong, Nak Jin Choi, Byung Hoon Kim, Yongseok Jun, and Hyung-Kun Lee, Scientific Reports , 5, 10904(2015), http://doi.org/10.1038/srep10904

23. Lianlian Chen, Yu Liu, Yang Zhao, Nan Chen, and Liangti Qu, Nanotechnology, 27(3), 032001(2015), http://doi.org/10.1088/0957-4484/27/3/032001

24. Bok Y. Ahn, Eric B. Duoss, Michael J. Motala, Xiaoying Guo, Sang-Il Park, Yujie Xiong, Jongseung Yoon, Ralph G. Nuzzo, John A. Rogers, Jennifer A. Lewis, Science, 323(5921),1590-3(2009) http://doi.org/10.1126/science.1168375

25. S. Gamerith, A. Klug, H. Scheiber, U. Scherf, E. Moderegger, E. J. W. List, Advanced Functional Materials, 17(16), 3111(2007).

26. T. Rivkin, C. J. Curtis, A. Miedaner, J. Alleman, D. L.Schulz, D. S. Ginley, Electrochemical Society Proceedings, 27, 80(2001).

27. D. Lupo , W. Clemens, S. Breitung, K. Hecker, in Applications in Organic and Printed Electronics: A Technology-Enabled Revolution (Ed: E. Cantatore ), Springer, Boston, MA, USA 2013 , pp. 1 - 26

28. H. Sirringhaus , T. Kawase , R. H. Friend, T. Shimoda, M. Inbasekaran , W. Wu , E. P. Woo, Science, 290, 2123(2000).

29. R. R. Sondergaard, M. Hösel, F. C. Krebs, Journal of Polymer Science, Part B, Polymer Physics, 51(1), 16(2013), https://doi.org/10.1002/polb.23192

30. Lin Gu, Shuan Liu, Haichao Zhao, and Haibin Yu, ACS Applied Materials \& Interfaces, 7(32), 17641(2015), http://doi.org/10.1021/acsami.5b05531

31. Shuai Wang, Min Yi, and Zhigang Shen, RSC Advances, 6(61), 56705(2016), http://doi.org/10.1039/c6ra10933k

32. X. Han, Y. Chen, H. Zhu, C. Preston, J. Wan, Z. Fang and L. Hu, Nanotechnology, 24(20), 205304(2013), http://doi.org/10.1088/0957-4484/24/20/205304

33. Wei Meng, Elizabeth Gall, Fuyou Ke, Zhouhao Zeng, Benjamin Kopchick, Raju Timsina and Xiangyun Qiu, The Journal of Physical Chemistry C, 119(36), 21135(2015), http://doi.org/10.1021/acs.jpcc.5b04102

34. Sasha Stankovich, Richard D. Piner, Xinqi Chen, Nianqiang Wu, Son Binh T. Nguyen, and Rodney S. Ruoff, Journal of Materials Chemistry, 16(2), 155(2006), http://doi.org/10.1039/b512799h

35. Xiaoping Shen, Lei Jiang, Zhenyuan Ji, Jili Wu, Hu Zhou, and Guoxing Zhu, Journal of Colloid and Interface Science, 354(2), 493(2011), http://doi.org/10.1016/j.jcis.2010.11.037

36. Kyoungho Min, Tae Hee Han, Joohoon Kim, Jiyoung Jung, Cheolsoo Jung, Soon Man Hong, and Chong Min Koo, Journal of Colloid and Interface Science, 383(1), 36(2012), http://doi.org/10.1016/j.jcis.2012.06.021

37. Yafei Fan, Yushan Liu, Qiang Cai, Yongzhao Liu, and Jianmin Zhang, Synthetic Metals, 162(21-22), 1815(2012), http://doi.org/10.1016/j.synthmet.2012.08.016

38. K. N. Amba Sankar, C. Sathish Kumar, and Kallol Mohanta, Materials Today: Proceedings, 18, 759(2019), http://doi.org/10.1016/j.matpr.2019.06.492

[RJC-6452/2021] 